\title{
A Brief Analysis of News Collecting and Editing Strategies of Public Health Emergencies from the Perspective of New Media
}

\section{Comparison of the SARS ${ }^{1}$ Epidemic and the COVID-19² Outbreak $^{-}$} Bingjie Shi*

Zhejiang Ocean University, Zhoushan 316022, China. E-mail: sharon_shi218@163.com

Abstract: Public health emergency, especially contagion such as the SARS epidemic in 2003 and the COVID-19 outbreak recently happened, has been one of greatest challenges in human beings' history. How the government handles the epidemic situation will deeply reflect its political system and executive ability, as well as the capacity of all sectors of society to deal with public emergencies. The emergence and continuous development of new media technologies have promoted the transformation of news gathering and editing methods. Because of the experience gathered in the news coverage during SARS, the response speed of new media was greatly accelerated with the media literacy and professional level improved, which matters a great deal to the balance between the opening of information and public opinion control. Thus, government is capable of carrying out controls in a more effective way. Based on the research in the two cases, this paper aims to discuss the changes in efficiency, content transparency and form diversification of news collection and edit, with the discovery of some imperfections exposed in new media, in order to propose targeted improving measures.

Keywords: New Media; News Collection and Edit Strategies; Public Health Emergencies

\section{Introduction}

During the outbreak, explosive information spread on platforms, such as Weibo, WeChat and Toutiao. Under the development of new media, patterns of news collection and edit have undergone unprecedented revolution. Since the current conditions, the health-related information requirements of the public have been rapidly increasing for the high degree of concern about topics of life and health. And that the improvement of new media technology in information collection and news production plays a key role in the control of public panic as well as the promotion of effective measures exercised by government. Therefore, it is of great significance to probe into news collection and edit strategies in this new age.

\section{New working environment for news collection and edit of public health emergencies}

Information carriers are no longer limited by time and space in this era dominated by new media. At the same time, news source tends to be increasingly diversified due to the advancement of internet technology and the improvement of media literacy of the public. This revolutionary transformation provides new atmosphere full of thoughts and inspirations for the modern news media in dealing with public health emergencies.

As for public health incident, it refers to "a sudden outbreak with unknown source that may cause serious damage ${ }^{1}$ Severe Acute Respiratory Syndrome

${ }^{2}$ Coronavirus Disease in 2019

Copyright (C) 2020 Bingjie Shi

doi: $10.18686 / \mathrm{mcs} . v 2 \mathrm{i} 2.1289$

This is an open-access article distributed under the terms of the Creative Commons Attribution Non-Commercial License (http://creativecommons. org/licenses/by-nc/4.0/), which permits unrestricted non-commercial use, distribution, and reproduction in any medium, provided the original work is properly cited. 
to public health, such as infectious diseases, food poisoning, occupational poisoning and some other severe public emergencies". ${ }^{[1]}$ It is not only a major threat to the public's safety, but also a test for government's emergency-managing capacity and the media's journalistic professionalism. In a manner of speaking, not only is public health closely related to every citizen's basic rights, but also exerted a huge influence on social stabilization as well as country's economic and political development.

Throughout the ages, China and even the whole world have already suffered from plenty of public health crises frequently. With the rapid development of modern media technology, nowadays the public's demands for information in the field of public heath has been increased dramatically. It can be also seen as the original reason to carry on the research of this paper.

\section{Analyses of the changes by comparing SARS \& COVID-19}

On December 30, 2019, Wuhan Health Committee reported the pneumonia with unknown cause. On January 8 , 2020, National Health Commission preliminarily confirmed that the pathogen of the epidemic in Wuhan emerged as a novel coronavirus. Since then, the epidemic had spread throughout the country and even towards overseas. This event has attracted great attention of Chinese society and international community.

In fact, there has been similar incident called SARS in Chinese history. The incident occurred in Guangdong Province in December in 2002 and spread to the whole country, southeast Asia and even the whole world. However, due to the lack of experience in news collection and edit of public health emergencies, Chinese government and media at that time did not take correct and efficient measures, which hinders the realization of the effect of news communication.

Compared with the SARS epidemic, news media center in COVID-19 event has shifted significantlythe mobile internet has become the main media for the public to obtain news information. It is found that the news collection and edit of such unexpected public events presents two major changes as follows:

\subsection{Become more timely and more efficient}

At the beginning of SARS, on account of government's repression, media's collective aphasia and tanglesome public's access to information, there existed two extreme attitudes, namely "exaggeration" and "ignorance" among the public, wasting more than four months and delaying the work of warning and controlling.

It was not until April 20, 2003, when Party Central Committee and State Council issued instructions, "The media should promptly report and publicize the information of SARS epidemic", that the press got started in full swing. The delay in news work and the absence of media voices resulted in numerous unnecessary casualties. ${ }^{[1]}$

Relatively high effectiveness of news collection and edit of COVID-19 epidemic at this time reflects the timeliness of new media, which is far better than that of SARS. At the first sight of the appearance of COVID-19, almost all mainstream media, such as CCTV news and People's Daily on Weibo and WeChat, even numerous We-medias never stopped the spread of relevant news. It has not only aroused widespread public concern, but also prompted the national government to take corresponding measures.

\subsection{More transparent content to protect the public's rights to know}

In SARS period, the inexperienced government concealed the truth for fear of public panic, which greatly hindered the process of news collection and edit. Otherwise, due to the limited level of technical conditions and many other restrictions, news editorial content became inaccurate and fogged, as if covered with layers of dust, which greatly weakened the effect of the news dissemination. It would lead to more serious consequences_-inadequate protective measures and weak public epidemic-preventing awareness, which exacerbated the continuous spread of SARS epidemic. The heavy loss in SARS event has aroused a stronger requirement for informative transparency and also greatly alarmed the national government and media.

Since the SARS outbreak, the national government has been accelerating the construction of relevant laws and regulations, and vigorously developing an open, transparent and timely reporting mechanism for the spread of the epidemic. From May 9 to April 20 in 2003, Chinese State Council has drafted, discussed and promulgated Regulations on Public Health Emergencies, with that Publicity Department of the Communist Party of China issued Implementation Opinions on Improving and Strengthening the News Release of Domestic emergencies. In 2008, the state formally 
implemented the Regulations of the People's Republic of China on the Disclosure of Government Information, which comprehensively expands the scope of disclosing information and further protects citizens' right to know ${ }^{[2]}$. In response, many media began to set up special columns on public health topics in order to address media's editorial problems of informative opacity exposed in SARS epidemic.

News report on COVID-19, by contrast, tends to be open and transparent. The public are able to see the updating data on the number of infections and death per day. They can also obtain the information which reveals the frontline situation in Wuhan and other hardest-hit areas through website. Beyond that, media nowadays use different methods like short videos, H5 or data map to visualize the real situation of the epidemic. One of the biggest advances in the news collection and compilation is to use big data technology to map the epidemic situation and to implement data across provinces, regions, communities and even households, which promotes the achievement of full-scale "information transparency". It guarantees citizens' rights to know, which hugely conduces to enhance the credibility of the media itself, and urges the government to carry out the epidemic preventing and controlling work more effectively.

\subsection{More diverse forms and closer interaction with the audiences}

Due to the monotonous types of media and means of dissemination during the SARS period, media's connection with the audiences were not strong enough, which greatly reduced the effect of news. At the same time, the framework and theme of news collection and edit stood not in a perfect and mature status. However, new media has formed their basic fixed structure of public health emergency news because of the improvement of the comprehensive newsproducing ability and media literacy of journalists and editors as well as the progress of information collecting and editing techniques, significantly optimizing the propagation effect. Good examples are reflected in mainstream media on Weibo platform. They use various methods to present news__dynamic updating of "Epidemic Map" mentioned above, instant updating short messages called "Epidemic Express" and short videos of frontline interviews. At the same time, many other We-medias and users also produce different sounds through various homemade short videos, pictures, short essays and comments, which provides a wider range of sources and references for news collection. In terms of news themes, each new media platform has set up more abundant sections with better framework. In addition to the special coverage related to the epidemic, People's Daily APP also established the section functioned "aid channel" on the top of the page to collect and transmit information of those who seek for help and promote the interaction with the audiences. Relevant sections on Weibo platform are inclined to be comprehensive. In addition to the real-time "top search" section, "fight against pneumonia" section has also been set up in an all-round way, including "Epidemic Map", "Wuhan Diary", "Overseas Daily”, "Epidemic Rumor Refuting” and so on. New media tend to collect the epidemic news from broader social channels, then to write and edit those news in a variety of ways.

\section{Problems and deficiencies of the contemporary media}

\subsection{Deleterious effects on progress in journalism caused by the spread of internet rumors}

Frequent occurrence of network rumors are triggered by several reasons including the uncertainty of network's "anonymity mechanism" and vague distinction between information transmitters and receivers. It does further affects the objective judgment of journalists and editors. Some rumors, after being unilaterally and wrongly interpreted, were even spread by mainstream media. For instance, at 22:54 on January 31, 2020, People's Daily released a message, "Shuanghuanglian may effectively suppress the new coronavirus", which results in a terrible shortage of Shuanghuagnlian oral liquid across the country.

From that, it can be seen that internet rumors seriously hinder the process of news production. Once rumors interfere with the specific operational process of journalists and editors, they will make news topics and themes deviate from the right direction. Meanwhile, rumors will waste editorial staff's time and energy, which lowers the efficiency of news production and real-time press release. It has not only led to misguidance, but expanded public's panic. Worst of all, it may affect the credibility of mainstream media.

\subsection{News reports with a lack of in-depth discussion on future prevention}

In respect of public health emergencies, those news reports published on new media platforms have been already 
greatly improved in timeliness and accuracy compared with the past. However, it is rare for media to comprehensively set the agenda on future prevention. There still exists a big blank in the area of epidemic precaution in days to come and the popularization of scientific knowledge about health protection. Instead of playing due role in guiding the public to deeply understand the truth under "iceberg" and attaching great importance to the issue of "future prevention", the vast majority of media focus more on collecting up-to-date information a00nd producing instant news in order to catch the quality of news at the present stage, new media in the future should think more about how to choose appropriate agenda and how to grasp the degree of news reports.

\subsection{Language ambiguity and cognitive differences on account of "Digital Divide"}

One of the popular definitions of digital divide is described by Dr. Penglan in her publication, Introduction to Network Communication. The "Digital Divide" refers to "Differences in the mastery and use of digital technologies in the Information Age due to different factors, such as geographic region, personal income, educational level, race etc, which may cause inequalities among different groups in society". ${ }^{[3]}$

Because of the differences in educational level and the application ability of digital media technology, some audiences are not good at searching information on new media platforms, nor can they correctly understand some official and professional news language. Meanwhile, many of media put themselves in a "high position", ignoring the audiences', especially the elderly's comprehensive ability and real needs, leading to the immense decrease of the effect of news dissemination. Once some audiences misunderstood with the situation that the information released by those We-medias often misses gatekeepers, it is liable to give rise to more rumors, which may finally lead to a vicious circle of information.

\section{Suggestions for news collection and edit of public health emergencies in the future}

\subsection{To confirm the fact from multidimensional sources and cooperate with traditional media}

Journalists and editors should always maintain scientific and objective, bewaring of being disturbed by network rumors. In addition, the purpose of news production is supposed to figured out with an establishment of an overall view. In order to realize the significance of news collection, the news-related staff should carry out multiple confirmation. And journalists need to confirm the accuracy of news sources and essential truth of news work with logical truth in allaround ways. They'd better not only ensure the facts, but also dig out the underwater truth of the news. Only to this extent can they better reflect the actual situation, meet the public's information requirements and further facilitate the control of public opinion.

Moreover, new media ought to cooperate with the authoritative traditional media. For new media, they tend to have faster information-gathering capabilities and more diverse methods in news edit. For traditional media, they tend to own more credibility. From this point, both traditional and new media can try to learn and draw on strengths from each other. Under mutual supervision and balance, they can work together to prevent the emergence and spread of rumors and finally make the contemporary media become more excellent in settling such matters.

\subsection{To optimize the journalistic mode in information gathering and news editing with proactive setting of media agenda}

Scientific plans for informative pre-collection and pre-edit of news occupy a crucial position in news production. Thus all the media, especially those opinion leaders, are expected to optimize the original and inherent mode of collection and edit as well as to set agenda actively for the sake of guiding the public opinions on topics such as future prevention of epidemics, public health and safety etc. New media should also make good use of its interaction with audiences so as to collect effective feedbacks and finally build interdependent relationship with them. After enhancing media credibility and establishing a close relation with audiences or users, it tends to become reality in the future that media are able to cultivate public health awareness and helps strengthen the ability for the whole society to respond when emergent public disease outbreaks.

In such age, media are more obliged to consider the long-term development of the country and develop skills of 
news collection and edit to convey the in-depth thinking and correct values of our society and our nation.

\subsection{To use down-to-earth language and pay more attention to audiences or users' voices}

Everyone is concerned about health and safety, for it covers almost all aspects of their social lives. In order to avoid the obstacles brought by the "Digital Divide", the language of news needs to write in the perspective of the public or edit in a readable and comprehensible way with emphasis on simplicity and humanity at multiple levels. Using more visualization techniques like data map, diagrams or short videos, for example, is a big step forward. In the future, how to combine different means of new media together and incorporate these techniques into news writing and editing skills will become a question worth to explore.

In order to realize the popularization of news language and maximize the effectiveness of news production, it is of great necessity to consider different levels of news sources. The mainstream media, in particular, need to balance the official information and the information from different parties in society, then build a new mode for news collection and edit, given the highly-centralized information and the single source of news.

It is also significant to pay attention to the voices of audiences or users in comments section or UGC part. On the one hand, the media should timely understand the basic even more specific information requirements of their audiences or users so as to grasp the direction of news production accurately. On the other hand, the media should make good use of UGC information, then acquire the health dynamics of each region in time, for the purpose of laying a good foundation in information gathering and subsequent health news transmission.

All in all, it is so clear to see the significance of health news communication during the Covid-19 epidemic that has brought great disaster to people in both domestic and overseas. We are pleased to witness the remarkable improvement of media in promoting the process of epidemic control, compared to that time before in SARS event. Along with the changes of age and advancement of journalism, the development of working skills in news activities never cease moving on and will not stop stepping forward.

\section{References}

1. Ma S. Research on the changes of Chinese public health report discourse after the founding of new China (in Chinese). Shanghai International Studies University 2014.

2. Xiong W. Research on the evolution of ideas, paradigms and countermeasures of public health incident reporting in China (in Chinese). Soochow University 2010.

3. Peng L. Introduction to Network Communication. Beijing: China Renmin University Press; 2012.

4. Shi L. Introduction to New Media (in Chinese). Beijing: Communication University of China Press; 2009.

5. Yuan H. On the new features of news acquisition and editing under the background of media fusion (in Chinese). Journal News Research 2019; 10(23): 142+144.

6. Ding H. Analysis of the influence of internet communication on news editing (in Chinese). TV Guide 2018; (13): 129.

7. Chen X. A study on the historical evolution of disaster reporting in "People's Daily" (in Chinese). Lanzhou University 2016.

8. Du Y. Research on reporting strategies of sudden public health incidents by People's Daily in the last ten years (in Chinese). Hubei University 2015.

9. Zhu J. A study on the openness of government information for public emergencies in China from 2003 to 2014 (in Chinese). Tianjin Normal University 2014.

10. Wulangerile. Some thoughts on news editing in the self-media era (in Chinese). New Media Research 2016; 2(4): $137-138$. 